\title{
Semi-Analytical model for predicting breathing in Metal-Organic Frameworks
}

\author{
L. Vanduyfhuys, A. Ghysels, S.M.J. Rogge, R. Demuynck, and V. Van \\ Speybroeck* \\ Center for Molecular Modeling (CMM), Ghent University, Technologiepark 903, 9052 \\ Ghent, Belgium, (Member of the QCMM Ghent-Brussels Alliance Group) \\ E-mail: Veronique.VanSpeybroeck@Ugent.be \\ Supporting Information
}

${ }^{*}$ To whom correspondence should be addressed 


\section{Contents}

1 Mean-field approximation $\quad 3$

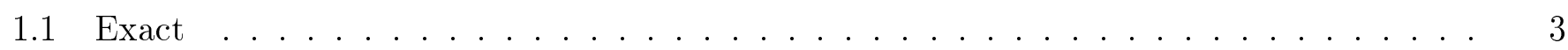

1.2 Rigid host framework . . . . . . . . . . . . . . . . . . . 4

1.3 Linearity in number of guest molecules . . . . . . . . . . . . . . . 5

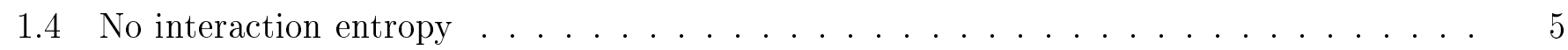

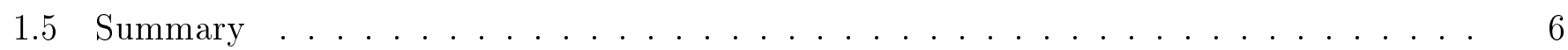

2 Thermodynamic limit of infinite number of unit cells $\quad 7$

3 Function $A(f) \quad 9$

3.1 Approach 1: point of inflection . . . . . . . . . . . . . . . 10

3.2 Approach 2: roots of third degree polynomial _. . . . . . . . . . . . 11

3.3 Solution of the chemical potential equation . . . . . . . . . . . . . . . 12

3.4 Relation with critical temperature of a van der Waals gas . . . . . . . . . . . . 12

4 Stability condition $\quad 14$

5 Parameterization of MIL-53(Al) input data $\quad 16$

6 Input parameters for $\mathrm{CH}_{4}$ and $\mathrm{CO}_{2}$ adsorption in MIL-53(Cr) 17

7 Input parameters for xenon adsorption in MIL-53(Al) 18 


\section{Mean-field approximation}

\subsection{Exact}

Consider the system of a metal-organic framework with adsorbed guest molecules at a temperature $T$. The total classical partition function of this system can be written as:

$$
Z=\int e^{-\beta U\left(\vec{q}_{h}, \vec{q}_{g}^{N}\right)} \overrightarrow{d q}_{h} \overrightarrow{d q}_{g}^{N}
$$

$\vec{q}_{h}$ represents the degrees of freedom of the host framework (i.e. the MOF), while $\vec{q}_{g}^{N}$ represents the degrees of freedom of $N$ guest molecules. These degrees of freedom include both all particle nuclear coordinates as well as the conjugate momenta. $U\left(\vec{q}_{h}, \vec{q}_{g}^{N}\right)$ represents the total internal energy, i.e. kinetic and potential energy, of the combined system. This energy is now split into three parts: a host internal energy, a guest internal energy and an interaction internal energy.

$$
U\left(\vec{q}_{h}, \vec{q}_{g}^{N}\right)=U_{\text {host }}\left(\vec{q}_{h}\right)+U_{\text {guest }}\left(\vec{q}_{g}^{N}\right)+U_{\text {int }}\left(\vec{q}_{h}, \vec{q}_{g}^{N}\right)
$$

At this point, no approximation has been made, this last equation just defines the interaction energy. The partition function becomes:

$$
\begin{aligned}
Z & =\int \overrightarrow{d q}_{h} \overrightarrow{d q}_{g}^{N} e^{-\beta U_{\text {host }}\left(\vec{q}_{h}\right)} e^{-\beta U_{\text {guest }}\left(\vec{q}_{g}^{N}\right)} e^{-\beta U_{\text {int }}\left(\vec{q}_{h}, \vec{q}_{g}^{N}\right)} \\
& =\left[\int \overrightarrow{d q} \vec{q}_{h} e^{-\beta U_{\text {host }}\left(\vec{q}_{h}\right)}\right] \cdot\left[\int \overrightarrow{d q}_{g}^{N} e^{-\beta U_{\text {guest }}\left(\vec{q}_{g}^{N}\right)}\right] \cdot\left[\frac{\int \overrightarrow{d q}_{h} \overrightarrow{d q}_{g}^{N} e^{-\beta U_{\text {host }}\left(\vec{q}_{h}\right)} e^{-\beta U_{\text {guest }}\left(\vec{q}_{g}^{N}\right)} e^{-\beta U_{\text {int }}\left(\vec{q}_{h}, \vec{q}_{g}^{N}\right)}}{\int \overrightarrow{d q}_{h} \overrightarrow{d q}_{g}^{N} e^{-\beta U_{\text {host }}\left(\vec{q}_{h}\right)} e^{-\beta U_{\text {guest }}\left(\vec{q}_{g}^{N}\right)}}\right]
\end{aligned}
$$

We give each contribution to the partition function the appropriate name:

$$
\begin{aligned}
Z_{\text {host }} & =\int \overrightarrow{d q}_{h} e^{-\beta U_{\text {host }}\left(\vec{q}_{h}\right)} \\
Z_{\text {guest }} & =\int \overrightarrow{d q}_{g}^{N} e^{-\beta U_{\text {guest }}\left(\vec{q}_{g}^{N}\right)} \\
Z_{\text {int }} & =\frac{\int \overrightarrow{d q}_{h} \overrightarrow{d q}_{g}^{N} e^{-\beta U_{\text {host }}\left(\vec{q}_{h}\right)} e^{-\beta U_{\text {guest }}\left(\vec{q}_{g}^{N}\right)} e^{-\beta U_{\text {int }}\left(\vec{q}_{h}, \vec{q}_{g}^{N}\right)}}{\int \overrightarrow{d q}_{h} \overrightarrow{d q}_{g}^{N} e^{-\beta U_{\text {host }}\left(\vec{q}_{h}\right)} e^{-\beta U_{\text {guest }}\left(\vec{q}_{g}^{N}\right)}}
\end{aligned}
$$


$Z_{\text {int }}$ can be interpreted as the average of $e^{-\beta U_{\text {int }}}$ in the ensemble generated by the non-interacting system with potential $U_{\text {host }}+U_{\text {guest }}$ :

$$
Z_{\text {host }}=\left\langle e^{-\beta U_{\text {int }}\left(\vec{q}_{h}, \vec{q}_{g}^{N}\right)} \mid U_{\text {host }}+U_{\text {guest }}\right\rangle
$$

At this point, all equations are still exact. We now proceed with the mean-field approximation. The three components of this approximation are:

1. The intermolecular interaction energy can be calculated with a rigid host framework.

2. The interaction free energy of $N$ guest molecules is equal to $N$ times the interaction free energy of one particle.

3. The interaction entropy of one particle is negligible.

\subsection{Rigid host framework}

We assume that the framework can be kept fixed for the calculation of the interaction part of the partition function. This would be a valid approximation whenever the potential energy of the total system increases much faster upon perturbing the framework atoms compared to perturbing the guest molecules. This is indeed the case, the framework atoms are bonded by means of strong covalent interactions, while the guest molecules only interact through weaker non-bonding interactions. As a consequence of this approximation, the framework nuclear coordinates in the interaction energy are replaced by a fixed set of coordinates, usually the equilibrium configuration $\vec{q}_{h}^{0}$. Hence, the interaction energy no longer depends on the framework coordinates which implies that the integration over the framework atoms in the numerator and denominator of Eq. 1.7 cancel out:

$$
\begin{aligned}
Z_{\text {int }} & =\frac{\int \overrightarrow{d q}_{g}^{N} e^{-\beta U_{\text {guest }}\left(\vec{q}_{g}^{N}\right)} e^{-\beta U_{\text {int }}\left(\vec{q}_{h}^{0}, \vec{q}_{g}^{N}\right)}}{\int \overrightarrow{d q_{g}^{N}} e^{-\beta U_{\text {guest }}\left(\vec{q}_{g}^{N}\right)}} \\
& =\left\langle e^{-\beta U_{\text {int }}\left(\vec{q}_{h}^{0}, \vec{q}_{g}^{N}\right)} \mid U_{\text {guest }}\right\rangle
\end{aligned}
$$

Hence, the interaction contribution can now be computed as an average of $e^{-\beta U_{\text {int }}}$ within a rigid framework in which the guest molecules are sampled according to their non-interacting potential $U_{\text {guest }}$. 


\subsection{Linearity in number of guest molecules}

Next, we assume that the particles can be assumed to not interact with each other when computing the interaction internal energy, in other words:

$$
\begin{aligned}
Z_{\text {int }}(N) & =\left[Z_{\text {int }}(1)\right]^{N} \\
F_{\text {int }}(N) & =-k_{B} T \ln Z_{\text {int }}(N) \\
& =N \cdot F_{\text {int }}(1)
\end{aligned}
$$

The expression for $Z_{\text {int }}(1)$ can be obtained from Eq. (1.9) by using the fact that for one particle, the guest energy vanishes $U_{\text {guest }}(1)=0$

$$
Z_{\text {int }}(1)=\int \overrightarrow{d q}_{g} e^{-\beta U_{\text {int }}\left(\vec{q}_{h}^{0}, \vec{q}_{g}\right)}
$$

Using standard formulas from statistical physics, we find:

$$
\begin{aligned}
F_{\text {int }}(1) & =-k_{B} T \ln Z_{\text {int }}(1)=E_{\text {int }}(1)-T \cdot S_{\text {int }}(1) \\
E_{\text {int }}(1) & =-\frac{\partial \ln Z_{\text {int }}(1)}{\partial \beta} \\
& =\frac{\int \overrightarrow{d q}_{g} U_{\text {int }}\left(\vec{q}_{h}^{0}, \vec{q}_{g}\right) e^{-\beta U_{\text {int }}\left(\vec{q}_{h}^{0}, \vec{q}_{g}\right)}}{\int \overrightarrow{d q}_{g} e^{-\beta U_{\text {int }}\left(\vec{q}_{h}^{0}, \vec{q}_{g}\right)}} \\
& =\left\langle U_{\text {int }}\left(\vec{q}_{h}^{0}, \vec{q}_{g}\right) \mid U_{\mathrm{int}}\right\rangle \\
& =\Delta U(V)
\end{aligned}
$$

This last line defines the interaction energy $\Delta U(V)$ used in the main article, it is dependent on the host unit cell volume through the interaction internal energy $U_{\text {int }}$ as well as the fixed framework coordinates $\vec{q}_{h}^{0}$.

\subsection{No interaction entropy}

The final approximation implies that a single particle has no interaction entropy, in other words $S_{\mathrm{int}}(1)=$ 0. 


\subsection{Summary}

Applying all three approximations, we end up with the following expression for the interaction free energy:

$$
\begin{aligned}
F_{\text {int }}(N) & =N \cdot F_{\text {int }}(1)=N \cdot E_{\text {int }}(1) \\
& =N \cdot \Delta U(V)
\end{aligned}
$$

Furthermore, the interaction internal energy contains only potential energy, no kinetic energy. Hence, the integration in $\Delta U$ also only runs over nuclear coordinates. This implies that in Eq. $(1.17), \vec{q}_{g}$ can be replaced by $\boldsymbol{r}$ (the Cartesian coordinates of a single guest molecule) and $\vec{q}_{h}$ can be replaced by $\boldsymbol{R}$ (the fixed coordinates of the rigid framework).

$$
\Delta U(V)=\frac{\int d \boldsymbol{r} U_{\mathrm{int}}(\boldsymbol{R}, \boldsymbol{r}) e^{-\beta U_{\mathrm{int}}(\boldsymbol{R}, \boldsymbol{r})}}{\int d \boldsymbol{r} e^{-\beta U_{\mathrm{int}}(\boldsymbol{R}, \boldsymbol{r})}}
$$




\section{Thermodynamic limit of infinite number of unit cells}

Let us investigate the dependence of the free energy on the number of unit cells $n$. Consider a framework supercell containing $n$ unit cells. The supercell volume is $n V$, the supercell pore volume is $n V_{p}(V)$ and the total number of particles adsorbed in this supercell is $n N$. The free energy of the empty host framework is linearly proportional to the amount of material, in other words to the number of unit cells.

$$
F_{\text {host }}(n, V, T)=n \cdot F_{\text {host }}(1, V, T)
$$

The interaction energy $\Delta U(V)$ represents the interaction energy of one adsorbed particle in one unit cell. Hence, the interaction free energy can actually be rewritten as:

$$
F_{\text {int }}(n, N, V, T)=n \cdot N \Delta U(V, T)
$$

The free energy of the guest molecules can be rewritten as:

$$
\begin{aligned}
F_{\text {guest }}(n, N, V, T) & =k_{B} T \ln (n N) !-n N k_{B} T \ln \left[\frac{P_{0}}{k_{B} T}\left(n V_{p}(V)-b n N\right)\right]-\frac{a(n N)^{2}}{n V_{p}(V)}+n N \mu_{0} \\
& =n\left[k_{B} T \frac{\ln (n N) !}{n}-N k_{B} T \ln \left[\frac{P_{0} b}{k_{B} T} \cdot n \cdot\left(\frac{V_{p}(V)}{b}-N\right)\right]-\frac{a(n N)^{2}}{n^{2} V_{p}(V)}+N \mu_{0}\right] \\
& =n\left[k_{B} T \frac{\ln (n N) !}{n}-N k_{B} T \ln n-N k_{B} T \ln \left(\frac{P_{0} b}{k_{B} T}\right)\right. \\
& \left.-N k_{B} T \ln \left(\frac{V_{p}(V)}{b}-N\right)-\frac{a N^{2}}{V_{p}(V)}+N \mu_{0}\right] \\
& =n\left[k_{B} T \frac{\ln (n N) !-n N \ln n}{n}-N k_{B} T \ln \left(\frac{V_{p}(V)}{b}-N\right)\right. \\
& -\frac{a N^{2}}{V_{p}(V)}+N \underbrace{\left(\mu_{0}-k_{B} T \ln \left(\frac{P_{0} b}{k_{B} T}\right)\right)}_{=\mu_{0}^{\prime}}] \\
& =n\left[k_{B} T \frac{\ln (n N) !-n N \ln n}{n}-N k_{B} T \ln \left(\frac{V_{p}(V)}{b}-N\right)-\frac{a N^{2}}{V_{p}(V)}+N \mu_{0}^{\prime}\right]
\end{aligned}
$$

$(n N)$ ! can be approximated using Stirling's formula, however, this is only valid for large values of $n N$. In the thermodynamic limit of very large number of unit cells $n$, but keeping a constant number of 
particles $N$ per unit cell and a constant volume $V$ per unit cell, the Stirling approximation is valid:

$$
\begin{aligned}
F_{\text {guest }}(n, N, V, T) & =n \cdot\left[k_{B} T \frac{n N \ln n N-n N-n N \ln n}{n}-N k_{B} T \ln \left(\frac{V_{p}(V)}{b}-N\right)-\frac{a N^{2}}{V_{p}(V)}+N \mu_{0}^{\prime}\right] \\
& =n \cdot\left[k_{B} T\left(N \ln \frac{n N}{n}-N\right)-N k_{B} T \ln \left(\frac{V_{p}(V)}{b}-N\right)-\frac{a N^{2}}{V_{p}(V)}+N \mu_{0}^{\prime}\right] \\
& =n \cdot\left[F_{\text {guest }}(1, N, V, T)\right]
\end{aligned}
$$

In this expression, $F_{\text {guest }}(1, N, V, T)$ is the single particle free energy of the Lennard-Jones gas assuming the Stirling approximation is valid. We now combine all contributions, and we drop the '1' from the argument list of the single particle free energies:

$$
\begin{aligned}
F(n, N, V, T) & =n \cdot\left[F_{\text {host }}(V, T)+F_{\text {guest }}(N, V, T)+F_{\text {int }}(N, V, T)\right] \\
& =n \cdot F(N, V, T)
\end{aligned}
$$




\section{Function $A(f)$}

The solution of the chemical potential equation is found by solving:

$$
\beta\left(\mu-\mu_{0}^{\prime}-\Delta U(V)\right)=A(f)
$$

in which a function $A$ was introduced:

$$
A(f):=\ln \frac{f}{1-f}+\frac{f}{1-f}-c f
$$

where the short notation $c=\frac{2 a}{k_{B} T b}$ was used for the dimensionless ratio of the van der Waals parameters. An important feature of this function was whether or not it is a monotonous function of $f$, because that will influence the solution greatly as was discussed in the main text. In this section, it is proven that

the function $A(f)$ will be monotonous for $f \in[0,1]$ if and only if $c<\frac{27}{4}$. A function is monotonic in an interval if its first order derivative does not change sign in that interval. This in turn means, that if the derivative is continuous in that interval, the derivative should not possess any zero in that interval. The first order derivative of $A$ is:

$$
\frac{d A}{d f}=\frac{1}{f(1-f)^{2}}-c
$$

The zeroes of this derivative are given by:

$$
\begin{array}{r}
\frac{d A}{d f}=\frac{1}{f(1-f)^{2}}-c=0 \\
f(1-f)^{2}-\frac{1}{c}=0 \\
f^{3}-2 f^{2}+f-\frac{1}{c}=0
\end{array}
$$

This is a third degree polynomial equation. There are two ways to proceed. One can use the general formula for the roots of a third-degree polynomial or one can reformulate the monotonous condition using the shape of the curve of $A(f)$. 


\subsection{Approach 1: point of inflection}

Let us first proceed using the latter approach. The curve of $A(f)$ is plotted in Fig. S1. The figure

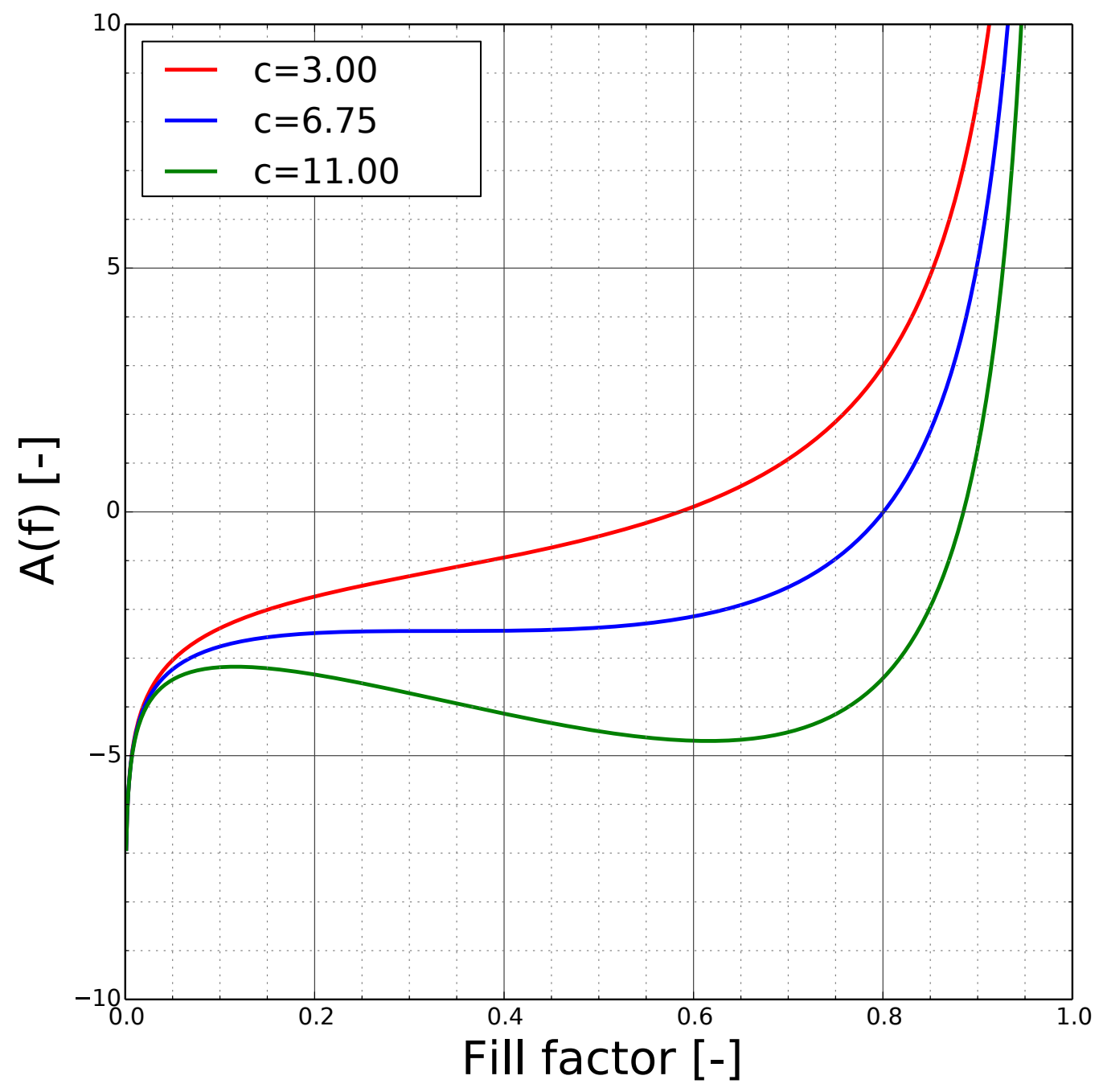

Figure S1: The function $A(f)$ for several values of $c$.

clearly illustrates that the curve has a point of inflection in the interval $[0,1]$. Furthermore, the function is a monotonous rising function when the slope at this inflection point is positive, while the function looses it monotonicity when the slope becomes negative at the inflection point. Hence, the condition of monotonicity can be reformulated as follows: the derivative at the inflection point should be nonnegative. This point of inflection is characterized with a zero in the second-order derivative:

$$
\frac{d^{2} A}{d f^{2}}=\frac{3 f-1}{f^{2}(1-f)^{3}}
$$


The inflection point is located at $f=\frac{1}{3}$. The slope of $A$ at this inflection point is then easily calculated as:

$$
\begin{aligned}
\frac{d A}{d f}\left(\frac{1}{3}\right) & =\frac{1}{\frac{1}{3}\left(1-\frac{1}{3}\right)^{2}}-c \\
& =\frac{27}{4}-c
\end{aligned}
$$

Finally we find:

$$
\begin{aligned}
\frac{d A}{d f}\left(\frac{1}{3}\right) & \geq 0 \\
c & \leq \frac{27}{4}
\end{aligned}
$$

\subsection{Approach 2: roots of third degree polynomial}

The second approach consists of investigating the roots of the third-order polynomial:

$$
f^{3}-2 f^{2}+f-\frac{1}{c}=0
$$

The number and nature (real-valued vs complex, single vs multiple) is determined by the determinant ${ }^{1}$ :

$$
\Delta=\frac{4}{c}-\frac{27}{c^{2}}
$$

When the determinant is positive, the polynomial has 3 single and real roots. When the determinant is zero, the polynomial has one double real root and one single real root. When the determinant is negative, the polynomial has one single real root and two single complex roots. Fig. 1 illustrates that the function $A$ has two extrema in the interval $[0,1]$ when it is no longer monotonous. Hence, for $A$ to be not monotonous, the polynomial in Eq. (3.12) should have at least 2 single and real valued roots. In other words, $A$ is not monotonous if the determinant is positive. Or reverse, for $A$ to be monotonous,

\footnotetext{
${ }^{1}$ The determinant of the polynomial $a x^{3}+b x^{2}+c x+d$ is $\Delta=18 a b c d-4 b^{3} d+b^{2} c^{2}-4 a c^{3}-27 a^{2} d^{2}$
} 
the determined should be negative:

$$
\begin{aligned}
\Delta=\frac{4}{c}-\frac{27}{c^{2}} & \leq 0 \\
c & \leq \frac{27}{4}
\end{aligned}
$$

\subsection{Solution of the chemical potential equation}

Figure 1 clearly illustrates that the behavior of the function $A(f)$ is dependent on the value of $c: A(f)$ is a monotonous function of the fill factor. This is only true if $c \leq \frac{27}{4}=6.75$, as is shown in the supporting information. This condition can always be achieved by increasing the temperature to a high enough value. In this case there is only one solution, which is stable. The systems in this work satisfy the condition $c \leq \frac{27}{4}$. If this condition is not satisfied, the inflection point of $A(f)$ has a negative slope, which means it is unstable. This inflection point is situated at a filling of one third $\left(f=\frac{1}{3}\right)$, which corresponds to the condition of condensation for a van der Waals gas. Furthermore, $A(f)$ then also has two extrema: a maximum $f_{M}$ in $] 0, \frac{1}{3}\left[\right.$ and a minimum $f_{m}$ in $] \frac{1}{3}, 1[$. This means that for certain values of $A$, Eq. (3.1) has 3 solutions: $f_{1}$ in $\left[0, f_{M}\right], f_{2}$ in $\left[f_{M}, f_{m}\right]$ and $f_{3}$ in $\left[f_{m}, 1\left[\right.\right.$. However only $f_{1}$

and $f_{3}$ are stable $\left(\frac{\partial^{2} \tilde{X}_{\mu P}}{\partial N^{2}}\right)$ and can hence be treated as thermodynamical valid solutions. They represent coexistency between the gas phase $\left(f_{1}\right)$ and the condensed phase $\left(f_{3}\right)$ of the guest molecules in the pores.

\subsection{Relation with critical temperature of a van der Waals gas}

The condition for monotonicity can be rewriten as follows:

$$
\begin{gathered}
c=\frac{2 a}{k_{B} T b} \leq \frac{27}{4} \\
T \geq T_{c}=\frac{8 a}{27 k_{B} b}
\end{gathered}
$$

Hence, the condition for monotonicity is equal to a temperature that is higher than the critical temperature $T_{c}$. Furthermore, the cricital point of a van der Waals gas also corresponds to a density of $\frac{N}{V}=\frac{1}{3 b}$, or in other words a fill factor of $f=\frac{N}{V b}=\frac{1}{3 b}$. Using Maxwell's area rule at a temperature below the critical temperature, we know that a van der Waals gas with a density within certain bounds of 
the critical density $\frac{1}{3 b}$ will split up in two phases, a liquid and a vapor, in equilibrium with each other. Due to the mean field approximation, our model predicts the same phase-splitting behavior of the guest molecules inside the pores below the critical temperature. 


\section{Stability condition}

As was mentioned in the main text, the double Legendre transform can either be done simultaneously or sequentially. Doing it simultaneously implies minimizing the $2 D$ function $\tilde{X}_{\mu P}(N, V)$ :

$$
\begin{aligned}
& \frac{\partial \tilde{X}_{\mu P}}{\partial N}=0 \\
& \frac{\partial \tilde{X}_{\mu P}}{\partial V}=0
\end{aligned}
$$

together with the stability condition, which says that the matrix of second-order derivatives evaluated in the solution should be positive definite. A matrix is positive definite if all eigenvalues are positive or, equivalently, if all leading principle minors ${ }^{2}$ are positive. In case of a $2 \times 2$ matrix this becomes:

$$
\begin{array}{r}
\frac{\partial^{2} \tilde{X}_{\mu P}}{\partial N^{2}}>0 \\
\left|\begin{array}{ll}
\frac{\partial^{2} \tilde{X}_{\mu P}}{\partial N^{2}} & \frac{\partial^{2} \tilde{X}_{\mu P}}{\partial N \partial V} \\
\frac{\partial^{2} \tilde{X}_{\mu P}}{\partial N \partial V} & \frac{\partial^{2} \tilde{X}_{\mu P}}{\partial V^{2}}
\end{array}\right|>0
\end{array}
$$

Doing the Legendre transform sequentially on the other hand, implies finding the minimum with respect to $N$ as a function of $V$ first, substituting the solution in $\tilde{X}_{\mu P}$ and finding the minimum with respect to $V$. In both minimization steps the stability condition needs to be verified:

$$
\begin{gathered}
\text { Step 1: } \frac{\partial \tilde{X}_{\mu P}}{\partial N}=0 \rightarrow N=N_{\mu}(V) \\
\frac{\partial^{2} \tilde{X}_{\mu P}}{\partial N^{2}}>0 \\
\text { Step 2: } \frac{d}{d V}\left(\tilde{X}_{\mu P}\left(N_{\mu}(V), V\right)\right)=0 \\
\frac{d^{2}}{d V^{2}}\left(\tilde{X}_{\mu P}\left(N_{\mu}(V), V\right)\right)>0
\end{gathered}
$$

\footnotetext{
${ }^{2} \mathrm{~A}$ leading principle minor is the determinant of an upper-left submatrix of the original matrix, i.e. the submatrix formed by the first $k$ rows and columns
} 
Both approaches are equivalent. In particular, both stability conditions are equivalent, which will now be proven. Eq. (4.8) can be rewriten as:

$$
\begin{aligned}
& \frac{d^{2}}{d V^{2}}\left(\tilde{X}_{\mu P}\left(N_{\mu}(V), V\right)\right)>0 \\
& \frac{d}{d V}\left(\frac{\partial \tilde{X}_{\mu P}}{\partial N} \frac{d N_{\mu}}{d V}+\frac{\partial \tilde{X}_{\mu P}}{\partial V}\right)>0 \\
& \frac{d}{d V}\left(\frac{\partial \tilde{X}_{\mu P}}{\partial V}\right)>0 \\
& \frac{\partial^{2} \tilde{X}_{\mu P}}{\partial N \partial V} \frac{d N_{\mu}}{d V}+\frac{\partial^{2} \tilde{X}_{\mu P}}{\partial V^{2}}>0
\end{aligned}
$$

The first term in Eq. (4.10) is zero because of the solution of the first step (Eq. 4.5), which means that the following identity holds:

$$
\frac{\partial \tilde{X}_{\mu P}}{\partial N}\left(N_{\mu}(V), V\right)=0
$$

This is an identity ${ }^{3}$, which means it holds for all unit cell volumes and we can drop it from Eq. (4.10). Furthermore, we can take the derivative of this identity towards $V$ :

$$
\begin{aligned}
\frac{d}{d V}\left(\frac{\partial \tilde{X}_{\mu P}}{\partial N}\left(N_{\mu}(V), V\right)\right) & =0 \\
\frac{\partial^{2} \tilde{X}_{\mu P}}{\partial N^{2}} \frac{d N_{\mu}}{d V}+\frac{\partial^{2} \tilde{X}_{\mu P}}{\partial N \partial V} & =0 \\
\frac{d N_{\mu}}{d V} & =-\frac{\frac{\partial^{2} \tilde{X}_{\mu P}}{\partial N \partial V}}{\frac{\partial^{2} \tilde{X}_{\mu P}}{\partial N^{2}}}
\end{aligned}
$$

Substituting this back in Eq. (4.12) gives:

$$
\begin{gathered}
\frac{\partial^{2} \tilde{X}_{\mu P}}{\partial N \partial V} \cdot\left(-\frac{\frac{\partial^{2} \tilde{X}_{\mu P}}{\partial N \partial V}}{\frac{\partial^{2} \tilde{X}_{\mu P}}{\partial N^{2}}}\right)+\frac{\partial^{2} \tilde{X}_{\mu P}}{\partial V^{2}}>0 \\
\frac{\frac{\partial^{2} \tilde{X}_{\mu P}}{\partial V^{2}} \cdot \frac{\partial^{2} \tilde{X}_{\mu P}}{\partial N^{2}}-\left(\frac{\partial^{2} \tilde{X}_{\mu P}}{\partial N \partial V}\right)^{2}}{\frac{\partial^{2} \tilde{X}_{\mu P}}{\partial N^{2}}}>0
\end{gathered}
$$

\footnotetext{
${ }^{3}$ The fact that it is an identity is important. It needs to hold for all unit cell volumes, otherwise the outer derivative towards $V$ could not be considered.
} 
Due to the stability condition of the first step, i.e. Eq. (4.6), the denominator is positive. This means we can finally write:

$$
\frac{\partial^{2} \tilde{X}_{\mu P}}{\partial V^{2}} \cdot \frac{\partial^{2} \tilde{X}_{\mu P}}{\partial N^{2}}-\left(\frac{\partial^{2} \tilde{X}_{\mu P}}{\partial N \partial V}\right)^{2}>0
$$

Which is equivalent with the second stability condition of the simultanous Legendre approach (Eq. 4.4).

\section{Parameterization of MIL-53(Al) input data}

All the required input data, i.e. the empty host free energy, the pore volume and the interaction energy, are calculated on a discrete volume grid. However, for the thermodynamical analysis, we require all the free energy contributions as analytical functions of volume. Therefore, the discrete grid data are fit to high-order Taylor expansions in terms of the unit cell volume. The host framework pressures obtained from the MD simulations were used to fit an $11^{\text {th }}$ order expansion in the volume. This pressure profile is then converted to a free energy profile using thermodynamic integration. The analytical expression for the pore volume profile was obtained by means of a $7^{\text {th }}$ order expansion in the volume using the

results from the Widom insertion simulations. Finally, an expansion in $\frac{1}{V}$ with exponents ranging from 6 to 16 in steps of 2 was used for the interaction energy. This particular choice was inspired by the functional form of the higher-order two-body dispersion series $\left(U_{\mathrm{disp}}(r)=\sum_{n=0}^{+\infty} \frac{C_{6+2 n}}{r^{6+2 n}}\right)$. 


\section{Input parameters for $\mathrm{CH}_{4}$ and $\mathrm{CO}_{2}$ adsorption in MIL-53(Cr)}

Fig. S2 summarizes the input required for the thermodynamic analysis of methane and carbon dioxide adsorption in MIL-53(Cr): the pore volume and interaction energy for methane and carbon dioxide in MIL-53(Cr) and the free energy of the empty framework of MIL-53(Cr). The numerical values of the van der Waals parameters and the Taylor expansion coefficients can be found in Ref. 1.
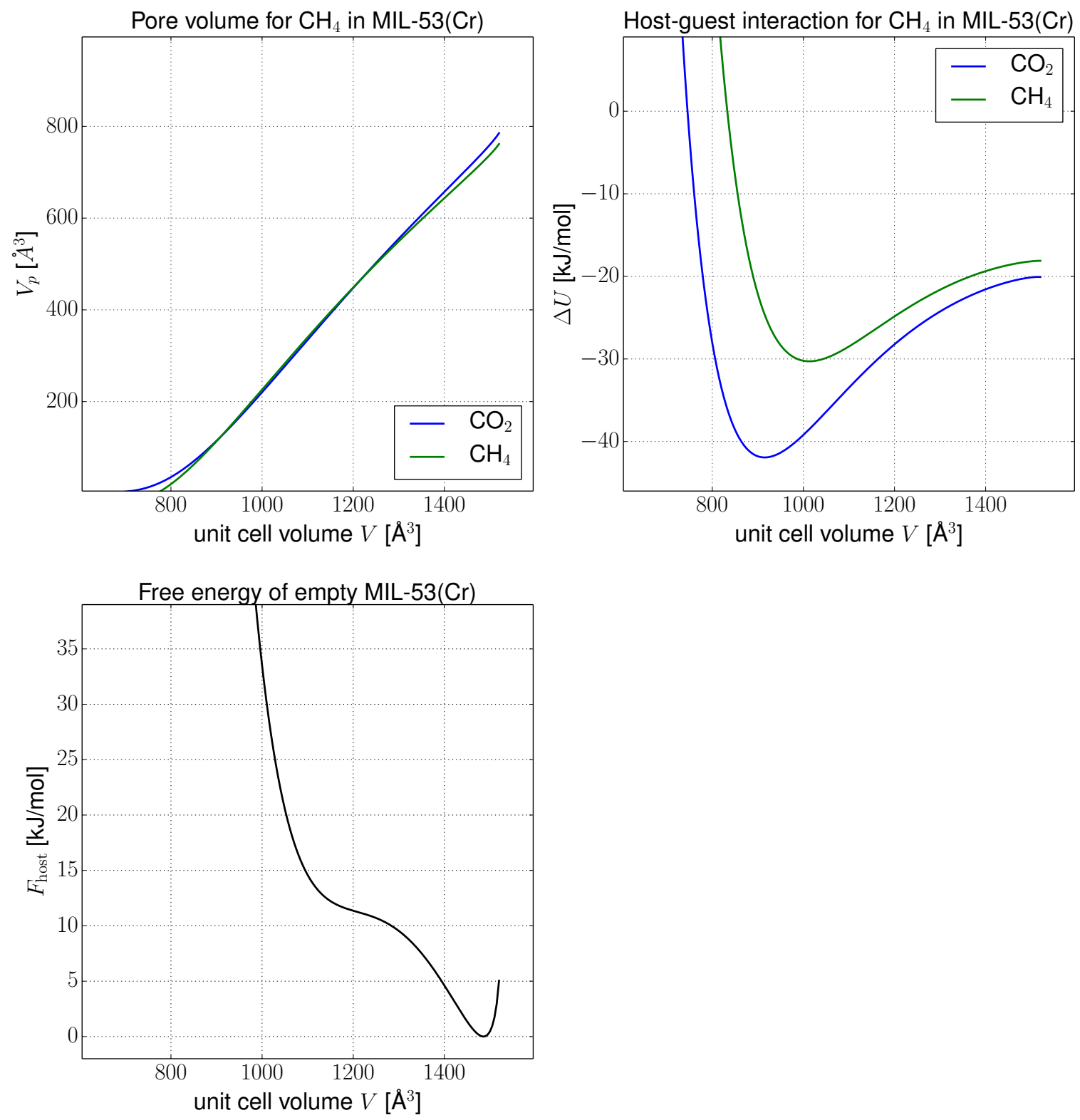

Figure S 2: Input for the thermodynamic analysis of methane and carbon dioxide adsorption in MIL$53(\mathrm{Cr})$. (top left) Pore volume for methane and carbon dioxide in MIL-53(Cr) (top right) Interaction energy for methane and carbon dioxide in MIL-53(Cr) (bottom left) Free energy of the empty MIL$53(\mathrm{Cr})$ framework 


\section{Input parameters for xenon adsorption in MIL-53(Al)}

Fig. 3 summarizes the input required for the thermodynamical analysis of xenon adsorption in MIL$53(\mathrm{Al})$ : the pore volume and interaction energy for xenon in MIL-53(Al) and the free energy of the empty framework of MIL-53(Al).
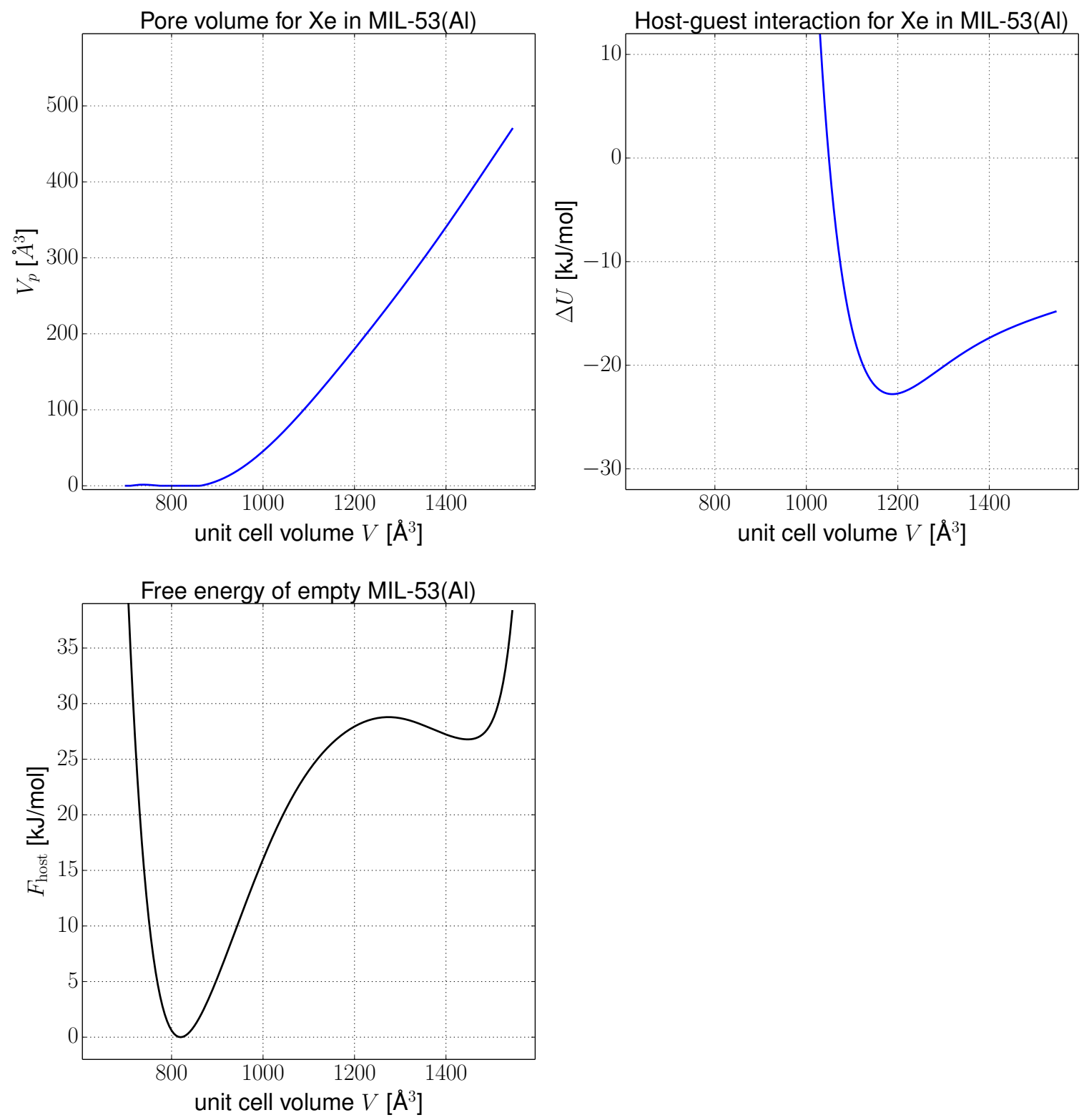

Figure S 3: Input for the thermodynamic analysis of xenon adsorption in MIL-53(Al). (top left) Pore volume for xenon in MIL-53(Al) (top right) Interaction energy for xenon in MIL-53(Al) (bottom left) Free energy of the empty MIL-53(Al) framework.

The van der Waals parameters of xenon are determined as followed: $b=84.771 \AA^{3}$ is taken from Ref. 2 and $a$ is determine such that the critical temperature of xenon is $285 \mathrm{~K}$, which results in $a=$ $677.951 \mathrm{~kJ} \mathrm{~mol}^{-1} \AA^{3}$. The Buckingham parameters of xenon were taken from the MM3 force field ${ }^{3}$ 
and transformed to Lennard-Jones parameters ${ }^{4}$. These LJ values, which were used in the Monte Carlo simulations, are $\sigma=4.06$ Aand $\epsilon=1.781 \mathrm{~kJ} \mathrm{~mol}^{-1}$. The Taylor expansions and their coefficients are given below.

$$
\begin{aligned}
F_{\text {host }}(V) & =k_{1} \cdot V^{1}+k_{2} \cdot V^{2}+k_{3} \cdot V^{3}+k_{4} \cdot V^{4}+k_{5} \cdot V^{5}+k_{6} \cdot V^{6} \\
& +k_{7} \cdot V^{7}+k_{8} \cdot V^{8}+k_{9} \cdot V^{9}+k_{10} \cdot V^{10}+k_{11} \cdot V^{11}+k_{12} \cdot V^{12} \\
\Delta U(V) & =k_{-16} \cdot V^{-16}+k_{-14} \cdot V^{-14}+k_{-12} \cdot V^{-12}+k_{-10} \cdot V^{-10}+k_{-8} \cdot V^{-8}+k_{-6} \cdot V^{-6} \\
V_{p}(V) & =k_{0} \cdot V^{0}+k_{1} \cdot V^{1}+k_{2} \cdot V^{2}+k_{3} \cdot V^{3}+k_{4} \cdot V^{4}+k_{5} \cdot V^{5}+k_{6} \cdot V^{6}+k_{7} \cdot V^{7}
\end{aligned}
$$

Table S1: Expansion coefficients of the Taylor expansion of the empty host free energy $F_{\text {host }}(V)$

$$
\begin{array}{cccc}
k_{1}\left[\mathrm{~kJ} \mathrm{~mol}^{-1} \AA^{-3}\right] & k_{2}\left[\mathrm{~kJ} \mathrm{~mol}^{-1} \AA^{-6}\right] & k_{3}\left[\mathrm{~kJ} \mathrm{~mol}^{-1} \AA^{-9}\right] & k_{4}\left[\mathrm{~kJ} \mathrm{~mol}^{-1} \AA^{-12}\right] \\
\hline 9.414 \cdot 10^{3} & -4.193 \cdot 10^{1} & 1.049 \cdot 10^{-1} & -1.572 \cdot 10^{-4} \\
k_{5}\left[\mathrm{~kJ} \mathrm{~mol}{ }^{-1} \AA^{-15}\right] & k_{6}\left[\mathrm{~kJ} \mathrm{~mol}{ }^{-1} \AA^{-18}\right] & k_{7}\left[\mathrm{~kJ} \mathrm{~mol}^{-1} \AA^{-21}\right] & k_{8}\left[\mathrm{~kJ} \mathrm{~mol}^{-1} \AA^{-24}\right] \\
\hline 1.302 \cdot 10^{-7} & -2.052 \cdot 10^{-11} & -8.358 \cdot 10^{-14} & 1.072 \cdot 10^{-16} \\
k_{9}\left[\mathrm{~kJ} \mathrm{~mol}{ }^{-1} \AA^{-27}\right] & k_{10}\left[\mathrm{~kJ} \mathrm{~mol}^{-1} \AA^{-30}\right] & k_{11}\left[\mathrm{~kJ} \mathrm{~mol}^{-1} \AA^{-33}\right] & k_{12}\left[\mathrm{~kJ} \mathrm{~mol}^{-1} \AA^{-36}\right] \\
\hline-6.813 \cdot 10^{-20} & 2.539 \cdot 10^{-23} & -5.303 \cdot 10^{-27} & 4.824 \cdot 10^{-31}
\end{array}
$$

Table S 2: Expansion coefficients of the Taylor expansion of the interaction energy $\Delta U(V)$

$$
\begin{array}{cccc}
k_{-16}\left[\mathrm{~kJ} \mathrm{~mol}^{-1} \AA^{48}\right] & k_{-14}\left[\mathrm{~kJ} \mathrm{~mol}^{-1} \AA^{42}\right] & k_{-12}\left[\mathrm{~kJ} \mathrm{~mol}^{-1} \AA^{36}\right] & k_{-10}\left[\mathrm{~kJ} \mathrm{~mol}^{-1} \AA^{30}\right] \\
\hline 6.637 \cdot 10^{51} & -2.705 \cdot 10^{46} & 4.454 \cdot 10^{40} & -3.670 \cdot 10^{34} \\
k_{-8}\left[\mathrm{~kJ} \mathrm{~mol}^{-1} \AA^{24}\right] & k_{-6}\left[\mathrm{~kJ} \mathrm{~mol}^{-1} \AA^{18}\right] & \\
\hline 1.532 \cdot 10^{28} & -2.707 \cdot 10^{21} &
\end{array}
$$

Table S 3: Expansion coefficients of the Taylor expansion of the pore volume $V_{p}(V)$

$$
\begin{array}{cccc}
k_{0}\left[\AA^{3}\right] & k_{1}\left[\AA^{0}\right] & k_{2}\left[\AA^{-3}\right] & k_{3}\left[\AA^{-6}\right] \\
\hline-4.534 \cdot 10^{4} & 2.759 \cdot 10^{2} & -7.003 \cdot 10^{-1} & 9.620 \cdot 10^{-4} \\
k_{4}\left[\AA^{-9}\right] & k_{5}\left[\AA^{-12}\right] & k_{6}\left[\AA^{-15}\right] & k_{7}\left[\AA^{-18}\right] \\
\hline-7.739 \cdot 10^{-7} & 3.654 \cdot 10^{-10} & -9.388 \cdot 10^{-14} & 1.012 \cdot 10^{-17}
\end{array}
$$

\footnotetext{
${ }^{4}$ imposing identical position and depth of the minimum
} 


\section{References}

(1) Ghysels, A.; Vanduyfhuys, L.; Vandichel, M.; Waroquier, M.; Van Speybroeck, V.; Smit, B. J. of Phys. Chem. C 2013, 117, 11540-11554.

(2) Weast. R. C. (Ed.), Handbook of Chemistry and Physics (53rd Edn.), Cleveland:Chemical Rubber Co., 1972.

(3) Allinger, N.; Lii, J.; Yuh, Y. J. Am. Chem. Soc. 1989, 111, 8551-8566. 